\title{
Limulus amoebocyte lysate test via an open-microcavity optical biosensor
}

Jonathan Scudder

Jing Yong Ye 


\title{
Limulus amoebocyte lysate test via an open-microcavity optical biosensor
}

\author{
Jonathan Scudder ${ }^{a, b}$ and Jing Yong $\mathrm{Ye}^{\mathrm{a}, *}$ \\ ${ }^{a}$ The University of Texas at San Antonio, Department of Biomedical Engineering, San Antonio, Texas, United States \\ ${ }^{\mathrm{b}}$ Texas Biomedical Research Institute, San Antonio, Texas, United States
}

\begin{abstract}
Almost since its discovery, Limulus amoebocyte lysate $(L A L)$ testing has been an important part of the pharmaceutical quality control toolkit. It allows for in vitro endotoxin testing, which has replaced tests using animals, such as using rabbits' thermal response to judge pyrogenicity of test samples, thus leading to a less expensive and faster test of parenteral pharmaceuticals and medical devices that contact blood or cerebrospinal fluid. However, limited by the detection mechanisms of the LAL assays currently used in industry, further improvement in their performance is challenging. To address the growing demand on optimizing LAL assays for increased test sensitivity and reduced assay time, we have developed an LAL assay approach based on a detection mechanism that is different from those being used in industry, namely, gel-clot, turbidimetric, and chromogenic detection. Using a unique open-microcavity photonic-crystal biosensor to monitor the change in the refractive index due to the reaction between LAL regents and endotoxins, we have demonstrated that this approach has improved the LAL assay sensitivity by 200 times compared with the commercial standard methods, reduced the time needed for the assay by more than half, and eliminated the necessity to incubate the test samples. This study opens up the possibility of using the significantly improved LAL assays for a wide range of applications. ๑ 2018 Society of Photo-Optical Instrumentation Engineers (SPIE) [DOI: 10.1117/1.JBO.23.2.027001]
\end{abstract}

Keywords: Limulus amoebocyte lysate; optical biosensor; endotoxin.

Paper 170638R received Sep. 30, 2017; accepted for publication Jan. 12, 2018; published online Feb. 6, 2018.

\section{Introduction}

Lipopolysaccharide (LPS), a naturally occurring endotoxin, is a structural component of Gram-negative bacteria cell membranes and is one of the best characterized pyrogens. Unfortunately, LPS is a very stable molecule, and it is difficult to remove from fluids or medications. In fact, the only way to remove the pyrogenic effects of LPS is to either denature it by high heat $\left(250^{\circ} \mathrm{C}\right.$ for $30 \mathrm{~min}$ or $200^{\circ} \mathrm{C}$ for $\left.1 \mathrm{~h}\right)$ or treatment with strong acids, bases, or hydrogen peroxide. Even low-pressure plasma sterilization, which has brought significant advances to laboratory equipment decontamination, does not adequately reduce the pyrogen load on a surface. ${ }^{1}$ If a parenteral drug or a medical implant has not been properly sterilized and contaminated with LPS, dramatic pathophysiological reactions may occur, including fever, leukopenia, tachycardia, tachypnea, hypotension, and disseminated intravascular coagulation. The septic shock can lead to multiorgan failure and a high mortality rate $(20 \%$ to $50 \%) .^{2}$ Therefore, it is critically important to have a sensitive approach for testing the endotoxin contamination.

Instead of using rabbit thermal response (i.e., fever) to a substance to judge its pyrogenicity, the Limulus amoebocyte lysate (LAL) test has been developed as an in vitro method for a faster and less-expensive approach, filling a sizeable need in the current pharmaceutical industry for quality control. ${ }^{3}$ The LAL test has become the most used assays of endotoxin owing to the pioneering work by Bang in 1956. While doing an unrelated experiment on horseshoe crab circulation, Bang and his colleague discovered that a crab had died due to systemic infection that caused its blood to almost completely gel. They determined that the gelation was due to an enzymatic cascade of the horseshoe crab monocyte, which reacted to the LPS in Gram-negative bacteria. LAL regents extracted from horseshoe crabs are currently being used in a huge variety of studies for endotoxin assays: testing bacterial growth in glaciers, ${ }^{4}$ testing for the presence of pyrogens in nanoparticle fluids, ${ }^{5}$ verifying safe work environments in ranching and farming, ${ }^{6}$ and observing that there are endotoxin and $(1 \rightarrow 3)-\beta$-D-glucan (a component of fungal cell walls) fluctuations with each season. ${ }^{7}$ Of course, the most important use of the LAL test, to date, is the testing of pharmaceutical contamination by Gram-negative bacteria. It is one of the few methods approved by the United States Pharmacopeia and FDA as an acceptable endotoxin testing strategy for oral and injectable medications, as well as implantable devices, such as in sepsis treatments, ${ }^{8}$ refining root canal drug delivery strategies, ${ }^{9,10}$ root canal procedure strategies, ${ }^{11}$ early indicators of intestinal membrane breakdown in dengue fever patients, ${ }^{12}$ and hip replacements, artificial hearts, and manmade ligaments. ${ }^{13}$ The LAL enzymatic cascade is one of the most sensitive enzymatic cascades known to man due to the fact that there are several amplifying steps that enhance the overall sensitivity of the test. The best commercial sources have a sensitivity of $0.005 \mathrm{EU} / \mathrm{mL}$, which equates to 0.0005 to $0.001 \mathrm{ng} / \mathrm{mL}$ for most bacterial endotoxins. ${ }^{14}$ The LAL gelclot reaction is a multistep enzymatic reaction that is initiated by the addition of the fluid of interest into the LAL assay solution in a 1:1 ratio. The endotoxin that may be in the fluid of interest then binds to factor $\mathrm{C}$ (it has also been shown that the endotoxin binds to both factors $\mathrm{C}$ and $\mathrm{B}$ initially), ${ }^{14}$ which then 
activates factor $\mathrm{B}$ and then activates the proclotting enzyme and cleaves the coagulogen protein, resulting in the gelation of the mixed solution.

In the most basic test- the end-point "gel-clot" test, one simply mixes the LAL assay standard solution with the analyte solution of interest in a $1: 1$ ratio at $37^{\circ} \mathrm{C}$. If after $1 \mathrm{~h}$ the combined solution gels, then the test is considered positive. In addition to the gel-clot test, there are two other approaches commercially available for LAL assays. The turbidimetric assay uses the same enzymatic cascade as the gel-clot test but adds a turbidity scanner to detect the change in scattered light over the whole reaction sequence, rather than only at the end-point to obtain kinetic analysis of the test sample. Chromogenic detection has also been developed as another approach for kinetic LAL assays. For that, the coagulogen in the final step of the enzymatic cascade is switched to a chromogenic substrate, which when cleaved by the activated clotting enzyme produces a yellow color that can be read in a standard plate reader.

Due to the limitations of the current LAL test, as well as the increasing demand of endotoxin testing in the pharmaceutical industry, refinements of pyrogen tests, including using Quartz sensors, ${ }^{15,16}$ electrochemical feedback sensors, ${ }^{17}$ nanoparticle suspensions, ${ }^{18}$ piezoelectric sensors, ${ }^{19}$ and surface-plasmonresonance (SPR)-based sensors, ${ }^{20}$ are constantly being made. In addition, alternative pyrogen test compounds, such as the beta glucan only test, Glucatell, ${ }^{21}$ the monoclonal antibody test, ${ }^{3,22}$ recombinant factor $\mathrm{C}^{4}$ and nanomolecular imprinted polymers, ${ }^{20}$ are constantly being engineered. However, each has inherent problems that result in them not being as sensitive or specific as the LAL test. In this paper, we report for the first time the development of a photonic-crystal total-internal-reflection (PC-TIR) biosensor for LAL assays, which utilizes a new mechanism to detect the reaction between LAL regents and analyte solutions via accurate monitoring of the change in the refractive index (RI) associated with the reaction. Our results have demonstrated the efficiency of this approach for rapid and highly sensitive LAL assays.

\section{Materials and Methods}

\subsection{Biosensor Chip Preparation}

The PC-TIR biosensor used in this work possesses a unique open optical microcavity for highly sensitive bioassays based on our patented detection mechanism of a photonic-crystal sensing technology. ${ }^{23,24}$ Different from our previous studies using the PC-TIR sensor for molecular binding assays, ${ }^{25-30}$ this study is focused on detecting the RI changes of LAL analyte solutions caused by endotoxins. The PC-TIR sensor was designed based on our numerical simulations with a transfer matrix approach and fabricated with electron-beam physical vapor deposition, which has five alternating 91-nm $\mathrm{TiO}_{2}$ and 309-nm $\mathrm{SiO}_{2}$ layers coated on a BK-7 glass substrate. Above this periodic structure, a cavity layer was formed with $382 \mathrm{~nm}$ of silica and $8 \mathrm{~nm}$ of silicon. The thin silicon layer was designed to give rise to an appropriate level of absorption such that a sharp dip in the reflectance spectrum is introduced at the resonant wavelength of an open optical microcavity formed when the PC structure is used in a TIR configuration. ${ }^{23,24}$ When the RI of analyte solutions on top of the PC-TIR sensor surface changes, the resonant wavelength shifts accordingly for $1490 \mathrm{~nm} / \mathrm{RIU}^{29}{ }^{29}$ Two sample wells were formed using a polydimethylsiloxane (PDMS) replica molding process. PDMS base and curing agents (Sylgard184, Dow Corning) were mixed at a ratio of 10:1. The mixture was degassed in a vacuum chamber for about $10 \mathrm{~min}$ and then cast on a mold and cured at room temperature. To bind the sample wells with the sensor chip, the surface of a PC-TIR sensor chip and the PDMS sample well were first processed with a plasma cleaner (from Harrick Plasma) for $60 \mathrm{~s}$, which renders the surface hydrophilic. The silanol $(\mathrm{SiOH})$ groups created on the surface form a bridging $\mathrm{Si}-\mathrm{O}-\mathrm{Si}$ bond when the oxidized PDMS surface is placed in contact with the sensor chip surface, creating an irreversible seal. The PC-TIR sensor chip together with the sample wells was baked at $200^{\circ} \mathrm{C}$ for $1 \mathrm{~h}$ before usage to remove any possible contaminations.

\subsection{Sensor Apparatus}

A lab-built sensor setup as shown in Fig. 1 was used to sensitively detect the changes in the RI of the LAL analyte solutions on the sensor chip surface. White light produced by a fiber illuminator (Thorlabs OSL1) was coupled into a single-mode optical fiber, and S-polarization was selected with a polarizer before the beam was split into two using a nonpolarizing beam splitter and mirrors. The light then passed to the sensor chip that was oriented in a TIR configuration, and the reflectance spectra of the two optical paths from the sensor were combined with a nonpolarizing beam combiner and detected with a high-resolution spectrometer (Ocean Optics HR 4000). Readings were taken via the Ocean View software package (Ocean Optics) and recorded as full spectrum data files every $30 \mathrm{~s}$. Each spectrum consisted of the average of 100.1 -s scans with boxcar smoothing with a radius of 2 .

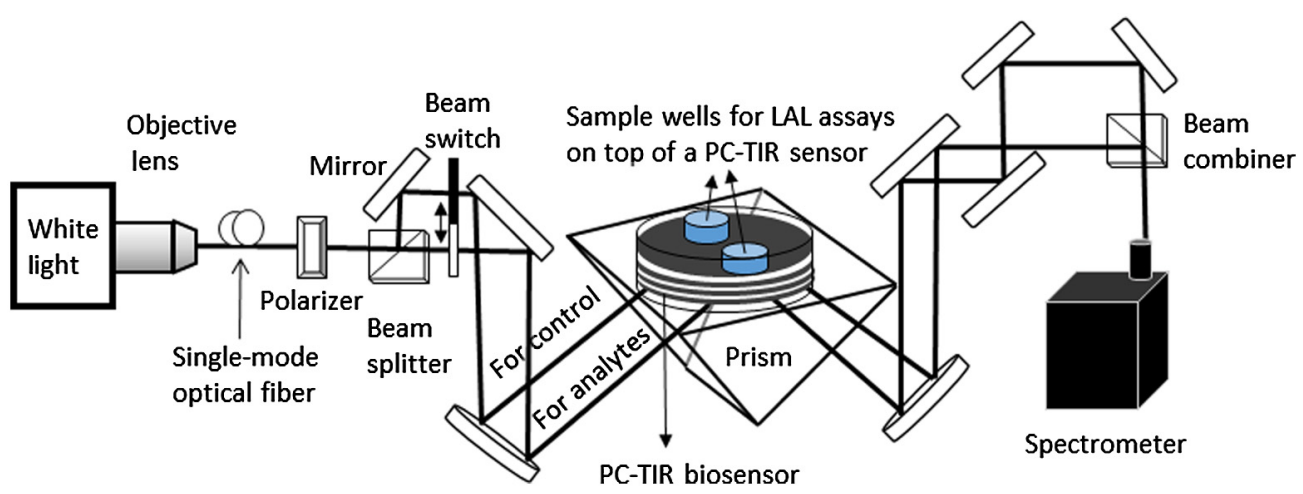

Fig. 1 Schematic drawing of the experimental setup for LAL assays using a PC-TIR biosensor. 


\subsection{Limulus Amoebocyte Lysate and Standard Endotoxin Preparation}

Charles River Laboratories supplied the LAL reagent KTA2 lot D2161L (0.005-EU/mL sensitivity). The directions for proper rehydration and preparation included in the LAL package were followed. The control standard endotoxin (CSE, Catalog \# E120) was also supplied by Charles River. Included in the CSE package was the Certificate of Analysis, which specifies the potency (pyrogenicity) of the dehydrated endotoxin in EU/mg. Solutions of 0.05, 0.0025, 0.00025, and $0.000025 \mathrm{EU} / \mathrm{mL}$ were made by dissolving the appropriate weight of endotoxin in LAL reagent water (LRW) (Charles River) according to the CSE instructions.

\subsection{Measurement Procedure}

After the appropriate dilutions of CSE were made and LAL reagents were rehydrated, the baked biosensor was placed on an equilateral prism with index matching fluid and $150 \mu \mathrm{L}$ of LRW was first added into both sample wells on the sensor. Alignment of the optical beam path reflected from the sensor was conducted to couple the probe beam into a high-resolution spectrometer so that resonant dips appeared at the correct wavelength in the reflectance spectrum of the sensor. The LRW was then replaced with $150 \mu \mathrm{L}$ of rehydrated lysate in the sample wells of the biosensor. Next, $150 \mu \mathrm{L}$ LRW was added into one well and the timer was started to record data in 30-s increments, while $150 \mu \mathrm{L}$ of the endotoxin sample was added into the other well. Detection of the spectrum of the reflected beams from the two wells was switched every $30 \mathrm{~s}$ so that the spectrometer measured the spectra of both wells every minute. Each run was monitored for $20 \mathrm{~min}$. After the assay, the sample wells and the sensor chip were cleaned first with acetone and then with deionized water and dried with compressed air to ensure that no fluid solution remained on the sensor surface. To reuse the biosensor, the cleaned sensor chip together with the sample wells was baked at $200^{\circ} \mathrm{C}$ on a hot plate or oven for at least $1 \mathrm{~h}$ in an aluminum enclosure, to ensure even heat transfer and to restrict room air contamination.

\subsection{Statistics}

Triplicates of all solutions were run to make statistical analysis possible. Because each data point consisted of the average of 10 spectral samples, the standard error was selected as the appropriate error bar in figures showing run results. Standard deviation and the coefficient of variation were also calculated using Excel. Adjusted $R^{2}$ values were used to judge the goodness of fit for the time-dependent data of the resonant wavelength shifts of a PC-TIR biosensor. Sensor drift was corrected for by subtracting the $\mathrm{H}_{2} \mathrm{O}$ only data from the other data sets.

\section{Results and Discussion}

In our previous studies, we used PC-TIR sensors for molecular binding assays ranging from well-studied coupling agents to small molecule binding and nucleic acid and cardiac biomarker detection. ${ }^{25-30}$ In contrast, this study, for the first time, utilizes the PC-TIR sensor to monitor the changes in the RI of LAL analyte solutions, which sensitively reflects the amount of endotoxin in the test samples. Figure 2 shows several representative reflectance spectra of a PC-TIR sensor at different time points

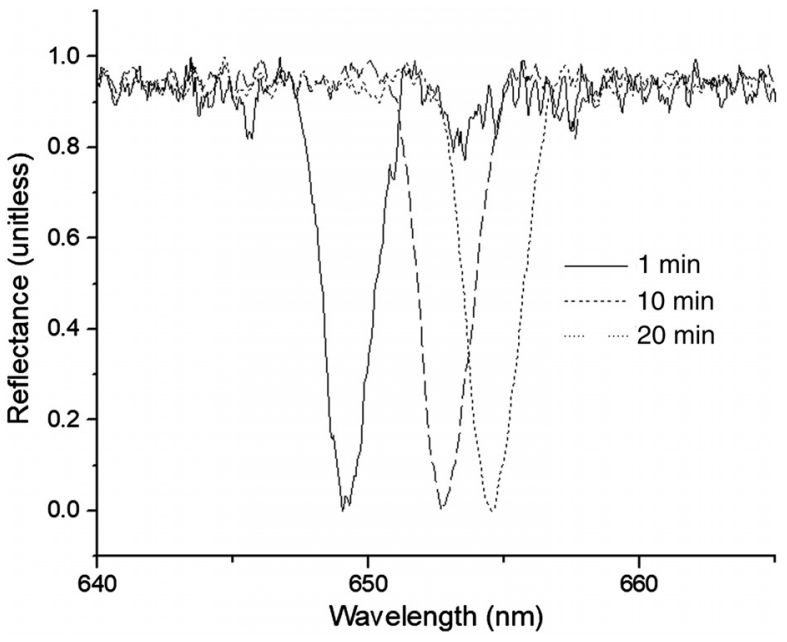

Fig. 2 Representative reflectance spectra of a PC-TIR biosensor with an LAL solution having $0.05-\mathrm{EU} / \mathrm{mL}$ endotoxin at different time points.

when an LAL analyte solution was added on the sensor surface. It can be seen that the sharp resonant dip shifts with time while the LAL responds to the endotoxin.

The wavelength shift of the resonant dip in the reflectance spectra of the PC-TIR biosensors was monitored with a highresolution spectrometer for every minute over a 20 -min period for each sample. The reflectance spectra were loaded into the OriginPro data analysis program to apply a Lorentzian curve fit and determine the center wavelength of the resonant dip. By fitting the curve rather than simply picking the lowest point of the spectrum, one can obtain a more accurate value of the resonant wavelength. The time dependence of the center resonant wavelength obtained from fitting the spectra is shown in Fig. 3. The results shown are for the test samples with different concentrations of endotoxin as well as the control sample.

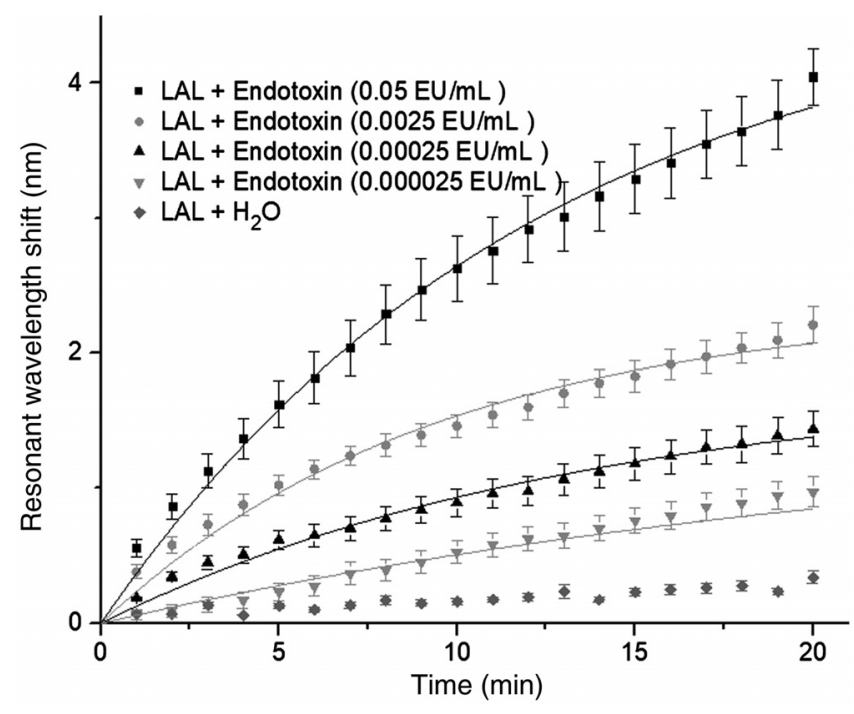

Fig. 3 The time dependence of the center wavelengths of the resonant dips in the reflectance spectra of a PC-TIR sensor for samples with different concentrations of endotoxin. The data are the results after subtracting the shift from a pure $\mathrm{H}_{2} \mathrm{O}$ sample to minimize any potential drift of the sensor. The error bars indicate the standard errors from different runs. The lines are from a nonlinear least squares fitting with a first-order exponential function in Eq. (1). 
The error bars represent the standard errors of different runs. The data indicate that even the most diluted endotoxin used $(0.000025 \mathrm{EU} / \mathrm{mL})$ resulted in a clearly measurable difference from the negative control (LAL with $\mathrm{H}_{2} \mathrm{O}$ ). Based on the average pyrogenicity of endotoxin, this equates approximately to $0.0000025 \mathrm{ng} / \mathrm{mL}$ of endotoxin in the solution, which is by far the greatest sensitivity of any previously published LAL testing strategies. Based on these results, we can see that the limiting factor is not the sensitivity of the LAL complex and it is the accurate and precise sensing of the gelation steps. This is further shown by the work of Noda et al. ${ }^{31}$ who increased the sensitivity of the LAL assay from 0.005 to $0.0005 \mathrm{EU} / \mathrm{mL}$ using an engineered luciferase that was 10 times brighter than the wild type. $^{32}$ As the LAL reagent obtained from Charles River Laboratories has a sensitivity of $0.005 \mathrm{EU} / \mathrm{mL}$, our result has demonstrated an enhancement of the detection sensitivity by 200-fold compared with the industry standard. Table 1 shows the comparison of our results with the methods used in the industry as well as those new approaches under development by different laboratories. ${ }^{15-17,19,20,31}$

The significant improvement of our approach in detection sensitivity can be attributed to the PC-TIR's capability for ultrasensitive measurements of the RI changes in the analyte solutions caused by LAL reactions to the minute amount of endotoxin. The sharp resonance bandwidth $(2 \mathrm{~nm})$ of the PCTIR sensor shown in Fig. 2 is much narrower than a typical SPR sensor $(\sim 40 \mathrm{~nm}),{ }^{33-35}$ thus allowing precise measurements of resonant wavelength shifts for enhanced detection sensitivity.

In addition to the sensitivity, the time required for LAL assays is also an important consideration in industry applications for higher throughput and cost saving. To illustrate the fast response of the PC-TIR sensor to LAL assays, we plotted the resonant wavelength shift of the sensor as a function of the endotoxin concentration at a selected time point, $5 \mathrm{~min}$, after endotoxins were added to LAL reagents. Figure 4 shows that the shift in the resonant wavelength of the sensor increases with increases of the concentration of the endotoxin, and within this short 5-min period, all the samples with different endotoxin concentrations can already be well distinguished. This result clearly demonstrates the fast response time, another unique

Table 1 A comparison of our results with the methods used in the industry as well as those new approaches under development by different research laboratories.

\begin{tabular}{lcc} 
Source & Sensitivity $(\mathrm{EU} / \mathrm{mL})$ & Response time (min) \\
\hline Ref. 15 & 0.001 & 100 \\
Ref. 16 & 0.005 & 50 \\
Ref. 17 & 0.01 & 60 \\
Ref. 19 & 0.001 & 40 \\
Ref. 20 & 0.0005 & Not given \\
Ref. 31 & 0.0005 & 15 \\
Charles River & 0.005 & $\begin{array}{c}60 \text { for gel-clot assay } \\
\text { and 15 for "Endosafe }\end{array}$ \\
& & Cartridge Technology" \\
Our approach & 0.000025 & 5 or less \\
\hline
\end{tabular}

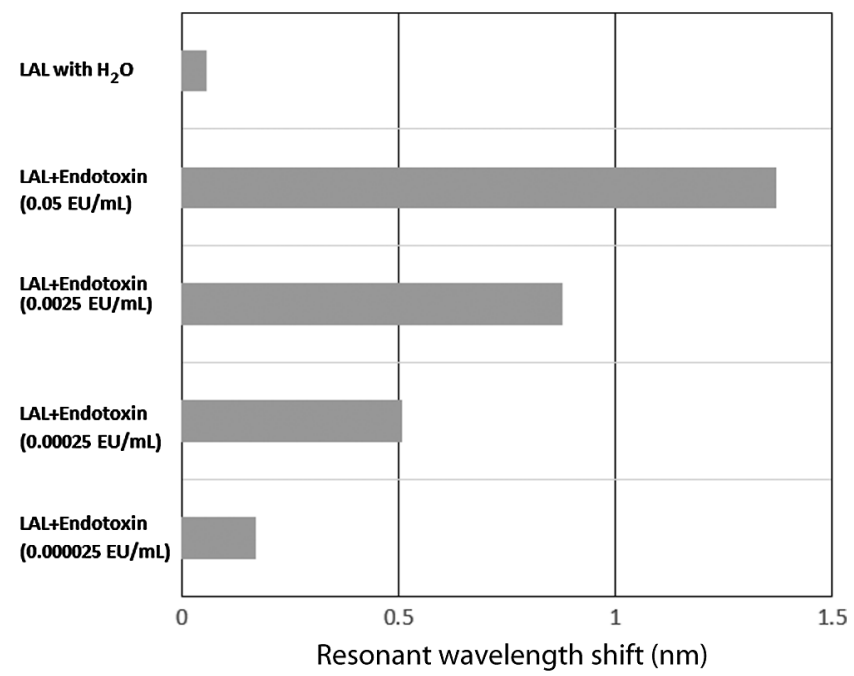

Fig. 4 Resonant wavelength shifts of a PC-TIR sensor at 5 min for test samples with different concentrations.

feature of the PC-TIR sensor-based LAL assays in addition to its ultrahigh sensitivity. It can be seen from Fig. 3 that different amounts of resonant wavelength shifts appear even as early as $2 \mathrm{~min}$, although we just selected the 5-min time point for a conservative claim. In contrast, the conventional approaches, including gel-clot, turbidimetric, and chromogenic assays, take a much longer time to generate assay results, especially for samples with low endotoxin concentrations. Gel-clot assays typical take $1 \mathrm{~h}$, while turbidimetric and chromogenic assays need about $15 \mathrm{~min}$ to complete one run. Even without the need for equilibrating to $37^{\circ} \mathrm{C}$, the fast response of the PC-TIR sensor can significantly shorten the assay time as shown in Table 1 in comparison with other methods, which is desired in many industry applications.

To obtain the reaction rate of the LAL assays detected with the PC-TIR sensor, we used a nonlinear least squares regression to analyze the time-dependent resonant wavelength shifts $\Delta \lambda$ of the sensor, which can be fitted to the following first-order exponential function:

$\Delta \lambda=A\left(1-e^{-B t}\right)$

where $A$ and $B$ are the fitting parameters. The fitting curves are plotted in Fig. 3, while the fitting parameters are listed in Table 2 for test samples with different endotoxin concentrations.

Table 2 Fitting results of the time-dependent resonant wavelength shifts for test samples with different concentrations of endotoxin.

\begin{tabular}{lcc} 
Sample & Fitting parameters + value & Adjusted $R^{2}$ \\
\hline $\begin{array}{l}\mathrm{LAL}+\text { endotoxin } \\
(0.05 \mathrm{EU} / \mathrm{mL})\end{array}$ & $\begin{array}{l}A=4.775 \pm 0.163 \\
B=0.081 \pm 0.005\end{array}$ & 0.994 \\
$\mathrm{LAL}+$ endotoxin & $A=2.355 \pm 0.088$ & \\
$(0.0025 \mathrm{EU} / \mathrm{mL})$ & $B=0.106 \pm 0.008$ & 0.984 \\
& & \\
$\mathrm{LAL}+$ endotoxin & $A=1.793 \pm 0.108$ & 0.964 \\
$(0.00025 \mathrm{EU} / \mathrm{mL})$ & $B=0.074 \pm 0.008$ & \\
$\mathrm{LAL}+$ endotoxin & $A=1.537 \pm 0.414$ & 0.958 \\
$(0.000025 \mathrm{EU} / \mathrm{mL})$ & $B=0.040 \pm 0.015$ & \\
\hline
\end{tabular}




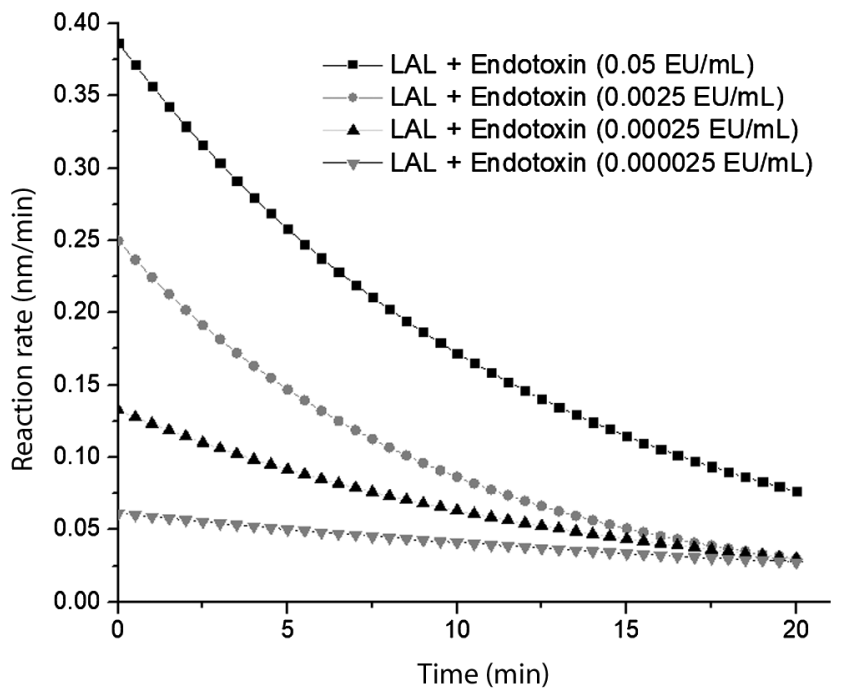

Fig. 5 Reaction rate between $\mathrm{LAL}$ and endotoxins with different concentrations.

The adjusted $R^{2}$ values obtained from the fitting are close to one, which indicates the goodness of fit. By taking the derivative of Eq. (1), we obtain the reaction rate as

Reaction rate $=A B e^{-B t}$,

which is plotted in Fig. 5. It can be seen that the reaction rate is generally higher for higher concentrations of endotoxin samples. Thus, the reaction rate at certain time points, for example at $t=0$, may also be utilized as a good parameter in addition to the resonant wavelength shifts to indicate endotoxin concentrations. Moreover, there is a general trend that the reaction rate for each sample decreases with assay time. This is reasonable as the reaction between LAL reagents and endotoxins reduces the amount of LAL reagents or free endotoxins and slows down the reaction.

\section{Conclusion}

We have shown for the first time that the LAL test with a PCTIR sensor provides value to the LAL assay in its significantly enhanced sensitivity by 200 -fold compared with the commercial standard methods, along with much faster discrimination of positive and negative results. The sensor chips can be reused as they are robust through a number heat cycles. This remarkable improvement can be attributed to the detection principle based on a PC-TIR sensor for sensitive monitoring of the RI changes in the analyte solutions, which is fundamentally different from other conventional methods of LAL assays. In addition, the PC-TIR sensor has a simple one-dimensional PC structure, which is basically a multilayered dielectric coating and can be easily fabricated with well-established vacuum deposition techniques. In contrast to two-dimensional or three-dimensional PC structures, the simplicity of the PC-TIR sensor structure allows for low-cost reproduction, robust performance, and yet ultrahigh sensitivity assays, which are important factors for potential commercialization of this unique technique for sensitive and rapid LAL assays.

Furthermore, there is promise in testing samples that possibly have confounding variables via extreme dilution due to the fact the LAL assays on a PC-TIR chip have a very high sensitivity.
Future research is greatly needed in this area, and a more complete list of confounding molecules should be tested with the PC-TIR chip with the endotoxin standard, blood plasma, and other analytes of interest. We also plan to investigate a method for binding the LAL proteins to the sensor surface, as this in conjunction with a microfluidic system would lead to lower volumes of LAL needed to test solutions for pyrogenicity.

\section{Disclosures}

The authors declare no conflicts of interest.

\section{Acknowledgments}

Charles River Laboratories graciously supplied the LAL reagents used in this study. This work was partially supported by the National Institutes of Health (No. R21 EB008765).

\section{References}

1. M. Moisan et al., "Low-temperature sterilization using gas plasmas: a review of the experiments and an analysis of the inactivation mechanisms," Int. J. Pharm. 226(1), 1-21 (2001).

2. J. Schletter et al., "Molecular mechanisms of endotoxin activity," Arch. Microbiol. 164(6), 383-389 (1995).

3. T. Hartung, "The human whole blood pyrogen test-lessons learned in twenty years," ALTEX: Altern. Anim. Exp. 32(2), 79-100 (2015).

4. M. J. Barnett et al., "In-field implementation of a recombinant factor C assay for the detection of lipopolysaccharide as a biomarker of extant life within glacial environments," Biosensors 2(1), 83-100 (2012).

5. S. Smulders et al., "Contamination of nanoparticles by endotoxin: evaluation of different test methods," Part. Fibre Toxicol. 9(1), 4141 (2012).

6. I. Basinas et al., "A comprehensive review of levels and determinants of personal exposure to dust and endotoxin in livestock farming," J. Exposure Sci. Environ. Epidemiol. 25(2), 123-137 (2015).

7. S. H. Hwang, C. S. Yoon, and J. B. Park, "Outdoor (1 $\rightarrow 3)$ - $\beta$-D-glucan levels and related climatic factors," J. Prev. Med. Public Health 47(2), 124-128 (2014).

8. M. H. Silverman and M. J. Ostro, Bacterial Endotoxin in Human Disease, XOMA (US) LLC, Berkeley, California (1999).

9. L. D. de Oliveira et al., "Efficacy of endodontic treatment for endotoxin reduction in primarily infected root canals and evaluation of cytotoxic effects," J. Endod. 38(8), 1053-1057 (2012).

10. A. C. Marinho, A. R. Polay, and B. P. Gomes, "Accuracy of turbidimetric Limulus amebocyte lysate assay for the recovery of endotoxin interacted with commonly used antimicrobial agents of endodontic therapy," J. Endod. 41(10), 1653-1659 (2015).

11. E. L. Sousa et al., "Quantification of endotoxins in infected root canals and acute apical abscess exudates: monitoring the effectiveness of root canal procedures in the reduction of endotoxins,' J. Endod. 40(2), 177-181 (2014).

12. C. A. van de Weg et al., "Lipopolysaccharide levels are elevated in dengue virus infected patients and correlate with disease severity," J. Clin. Virol. 53(1), 38-42 (2012).

13. N. Taylor, "The FDA and USP extend relationship to modernise drug quality," https://www.in-pharmatechnologist.com/Article/2011/05/ 10/FDA-USP-extend-relationship-to-modernise-drug-quality, (16 May 2011).

14. Y. Kobayashi et al., "Factor B is the second lipopolysaccharide-binding protease zymogen in the horseshoe crab coagulation cascade," J. Biol. Chem. 290(31), 19379-19386 (2015).

15. A. Chałupniak et al., "Application of quartz tuning forks for detection of endotoxins and Gram-negative bacterial cells by monitoring of Limulus amebocyte lysate coagulation," Biosens. Bioelectron. 58, 132-137 (2014).

16. T. Liu et al., "A quartz crystal microbalance sensor for endotoxin assay by monitoring Limulus amebocyte lysate protease reaction," Anal. Chim. Acta 961, 106-111 (2017). 
17. K. Y. Inoue et al., "Development of an electrochemical Limulus amebocyte lysate assay technique for portable and highly sensitive endotoxin sensor," Innate Immun. 18(2), 343-349 (2012).

18. Y. Li et al., "Optimising the use of commercial LAL assays for the analysis of endotoxin contamination in metal colloids and metal oxide nanoparticles," Nanotoxicology 9(4), 462-473 (2015).

19. H. Muramatsu et al., "Viscosity monitoring with a piezoelectric quartz crystal and its application to determination of endotoxin by gelation of Limulus amebocyte lysate," Anal. Chim. Acta 215, 91-98 (1988).

20. W. Su and X. Ding, "Methods of endotoxin detection," J. Lab. Autom. 20(4), 354-364 (2015).

21. T. Obayashi et al., "Plasma $(1 \rightarrow 3)$ - $\beta$-D-glucan measurement in diagnosis of invasive deep mycosis and fungal febrile episodes," Lancet 345(8941), 17-20 (1995).

22. I. Sander et al., "Development of a two-site enzyme immunoassay based on monoclonal antibodies to measure airborne exposure to $(1 \rightarrow 3)-\beta-D-$ glucan," J. Immunol. Methods 337(1), 55-62 (2008).

23. J. Y. Ye et al., "Analytical system with photonic crystal sensor," Patent No. 8,497,992 (2013).

24. J. Y. Ye et al., "Novel photonic crystal sensor," Patent No. 7,639,362 (2009).

25. B. Zhang et al., "Label-free detection of cardiac troponin I with a photonic crystal biosensor," Biosens. Bioelectron. 58, 107-113 (2014).

26. B. Zhang et al., "Detection of myoglobin with open-cavity-based labelfree photonic crystal biosensor," J. Med. Eng. 2013, 808056 (2013).
27. B. Zhang et al., "Detection of anthrax lef with DNA-based photonic crystal sensors," J. Biomed. Opt. 16(12), 127006 (2011).

28. Y. Guo et al., "Real-time biomolecular binding detection using a sensitive photonic crystal biosensor," Anal. Chem. 82, 5211-5218 (2010).

29. Y. B. Guo et al., "Sensitive molecular binding assay using a photonic crystal structure in total internal reflection," Opt. Express 16(16), 11741-11749 (2008).

30. B. Zhang et al., "Study of the interactions of Fusarium virguliforme toxin FvTox1 with synthetic peptides by molecular simulations and a label-free biosensor," Anal. Chem. 88(6), 3024-3030 (2016).

31. K. Noda et al., "Endotoxin assay by bioluminescence using mutant firefly luciferase," Anal. Biochem. 397(2), 152-155 (2010).

32. L. R. Stromberg, H. M. Mendez, and H. Mukundan, "Detection methods for lipopolysaccharides: past and present," Chapter 8 in Escherichia coli-Recent Advances on Physiology, Pathogenesis and Biotechnological Applications, A. Samie, Ed., InTech, Rijeka (2017).

33. J. Homola, "Surface plasmon resonance sensors for detection of chemical and biological species," Chem. Rev. 108(2), 462-493 (2008).

34. J. Homola, "Present and future of surface plasmon resonance biosensors," Anal. Bioanal. Chem. 377(3), 528-539 (2003).

35. X. D. Hoa, A. G. Kirk, and M. Tabrizian, "Towards integrated and sensitive surface plasmon resonance biosensors: a review of recent progress," Biosens. Bioelectron. 23(2), 151-160 (2007).

Biographies for the authors are not available. 